\title{
BMJ Open Patient and organisational variables associated with pressure ulcer prevalence in hospital settings: a multilevel analysis
}

\author{
Ida Marie Bredesen, ${ }^{1}$ Karen Bjøro, ${ }^{1}$ Lena Gunningberg, ${ }^{2}$ Dag Hofoss ${ }^{3}$
}

To cite: Bredesen IM, Bjøro K, Gunningberg L, et al. Patient and organisational variables associated with pressure ulcer prevalence in hospital settings: a multilevel analysis. BMJ Open 2015;5:e007584. doi:10.1136/bmjopen-2015007584

- Prepublication history for this paper is available online. To view these files please visit the journal online (http://dx.doi.org/10.1136/ bmjopen-2015-007584)

Received 5 January 2015 Revised 31 July 2015 Accepted 3 August 2015

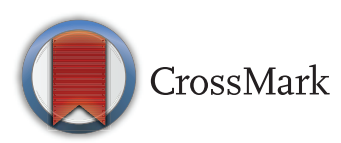

${ }^{1}$ Department of Orthopaedic Surgery, Oslo University Hospital, Oslo, Norway ${ }^{2}$ Department of Public Health and Caring Sciences, Caring Sciences, Uppsala University, Uppsala, Sweden

${ }^{3}$ Institute of Health and Society, University of Oslo, Oslo, Norway

Correspondence to Dr Ida Marie Bredesen; i.m.bredesen@medisin.uio.no

\section{ABSTRACT}

Objectives: To investigate the association of wardlevel differences in the odds of hospital-acquired pressure ulcers (HAPUs) with selected ward organisational variables and patient risk factors.

Design: Multilevel approach to data from 2 crosssectional studies.

Settings: 4 hospitals in Norway were studied.

Participants: 1056 patients at 84 somatic wards.

Primary outcome measure: HAPU.

Results: Significant variance in the odds of HAPUs was found across wards. A regression model using only organisational variables left a significant variance in the odds of HAPUs across wards but patient variables eliminated the across-ward variance. In the model including organisational and patient variables, significant ward-level HAPU variables were ward type (rehabilitation vs surgery/internal medicine: OR 0.17 ( $95 \% \mathrm{Cl} 0.04$ to 0.66$)$ ), use of preventive measures (yes vs no: OR 2.02 (95\% $\mathrm{Cl} 1.12$ to 3.64$)$ ) and ward patient safety culture (OR $0.97(95 \% \mathrm{Cl} 0.96$ to 0.99$)$ ). Significant patient-level predictors were age $>70$ vs $<70$ (OR $2.70(95 \% \mathrm{Cl} 1.54$ to 4.74$)$ ), Braden scale total score (OR $0.73(95 \% \mathrm{Cl} 0.67$ to 0.80$)$ ) and overweight (body mass index $\left.25-29.99 \mathrm{~kg} / \mathrm{m}^{2}\right)$ (OR $0.32(95 \% \mathrm{Cl}$ 0.17 to 0.62$)$ ).

Conclusions: The fact that the odds of HAPU varied across wards, and that across-ward variance was reduced when the selected ward-level variables entered the explanatory model, indicates that the HAPU problem may be reduced by ward-level organisation of care improvements, that is, by improving the patient safety culture and implementation of preventive measures. Some wards may prevent pressure ulcers better than other wards. The fact that ward-level variation was eliminated when patient-level HAPU variables were included in the model indicates that even wards with the best HAPU prevention will be challenged by an influx of high-risk patients.

\section{INTRODUCTION}

Organisational culture is a critical factor for successful implementation of quality improvement and development of patient safety

\section{Strengths and limitations of this study}

- This study contributes to research of the association between pressure ulcers and patient safety culture.

- The study was conducted in a single Regional Health Authority, which may reduce the generalisability of the findings.

- The study sample limits the number of variables included in the analysis.

culture. ${ }^{1}{ }^{2}$ Safety culture is often defined as the product of individual and group values, attitudes, competencies and patterns of behaviour that determine the commitment to, and the style and proficiency of, an organisation's health and safety programmes. ${ }^{3} 4$ Safety culture involves leadership, teamwork, shared belief in the importance of safety and learning. ${ }^{4}$

Quality and safety have become important healthcare policy objectives in many countries. ${ }^{2}$ In Norway, a patient safety campaign was initiated in 2011 that embraces a number of adverse events including pressure ulcer (PU) prevention, commonly considered an indicator of nursing care quality. A recent study in Norwegian hospitals found a PU prevalence of $18 \%$, a finding equal to or higher than prevalence rates in other European countries. ${ }^{5}$ This result is particularly disturbing considering that Norway has the highest expenditure on healthcare among European countries. ${ }^{6}$ Moreover, Norwegian hospitals were reported to have the lowest patient-to-nurse ratio in a large multicountry study in Europe, ${ }^{7}$ although a single-country analysis for Norway did show variation in staffing ratios across Norwegian hospitals. ${ }^{8}$ Still, the bottom line is that higher expenditure and greater number of nurses do not necessarily guarantee highquality or safe patient care. 
Despite extensive research and increased knowledge regarding patient-related PU risk factors and increased availability of evidence-based guidelines on PU prevention, the prevalence and incidence of PU have often proved resistant to change efforts. ${ }^{9-11}$ Many organisational factors, such as ward safety culture, could inhibit change. Some studies suggest that there is a link between stronger patient safety culture and lower PU rate. $^{12} 13$ Taylor et al ${ }^{12}$ found lower scores for patient safety domains in units with adverse events (patient falls, $\mathrm{PU}$, pulmonary embolism/deep vein thrombosis) than in units without. However, other studies have found no association between PU and organisational factors such as patient safety climate, team climate and preventive quality management at ward level. ${ }^{14}{ }^{15}$ Skin care, frequent repositioning, elevated heels and allocation of pressure redistributing mattresses are important nursing interventions to prevent $\mathrm{PU}$ according to an evidencebased PU guideline. ${ }^{11}$ Still, a large European study found a rather high prevalence of nursing tasks left undone, including documentation, skin care and repositioning due to lack of time, poor staffing levels and poor work environment. ${ }^{16}$ Increased productivity demands have led to greater patient turnover rates, leaving more tasks to be performed in less time, often by fewer staff. Further, the increased number of older patients and the increased prevalence of obesity and diabetes will probably lead to increased prevalence of PU. ${ }^{17}$

Studies of how organisational factors at ward level affect hospital-acquired PU (HAPU) prevalence have produced inconsistent results, ${ }^{12-15}$ indicating a need for further research. ${ }^{18}$ Moreover, policymakers at all levels are seeking research results to better understand how the quality of healthcare can be improved. ${ }^{19}$ The aim of this study was to study, within a multilevel statistical framework, the partition of the variance in the odds of HAPU into ward-level variance and patient-level variance, and investigate the association of selected ward organisational variables and patient risk factors on across-ward differences in HAPU odds in a sample of Norwegian hospitals.

\section{METHODS}

\section{Design}

This study uses two cross-sectional data sets collected from four Norwegian hospitals. One thousand and fiftysix patients from 84 somatic wards were included.

The patient safety culture data were obtained from a study conducted in all Norwegian Regional Health Authorities (RHAs) as part of the national patient safety campaign in Spring 2012. ${ }^{20}$ All health personnel at all hospitals in the country were asked to participate and complete a web-based questionnaire. Data were collected anonymously. The researchers were given the results, aggregated by ward, as written reports from the hospitals.

The PU prevalence study was conducted in voluntarily participating hospitals in the South-Eastern RHA in
October 2012. Inclusion criteria for this study were inpatients 18 years or above in somatic wards. Day surgery, psychiatric, maternity and paediatric wards were excluded from the study because of the low frequency of PU in such units. ${ }^{21}$ We excluded the patients with a PU at hospital admission as well as those patients with missing data for the PU present at hospital admission variable in the current study. The wards were surgery, internal medicine, rehabilitation and intensive care units (ICUs) (including postanaesthesia recovery). The data collection procedure for the PU prevalence study was the European Pressure Ulcer Advisory Panel's (EPUAP) methodology and trained nurse teams collected the data. The procedure has been described in greater detail elsewhere. ${ }^{5}$ Furthermore, the ward management completed an additional form including the number of patient beds on the ward, the number of staff and skill mix on each shift on the day prior to the prevalence study data collection and the number of inpatients at 07:00 on the prevalence study day.

\section{Measures}

The main outcome variable in this study was the prevalence of HAPU categories I-IV (table 1). The data collection teams identified patients admitted with a PU from the hospitals' patient record admission notes. In this study, HAPUs were defined as PUs not documented at hospital admission. HAPUs were classified according to the international classification: category I: nonblanchable erythema, category II: partial thickness skin loss, category III: full thickness skin loss, and category IV: full thickness tissue loss including also unstageable and suspected deep tissue injury. ${ }^{11}$

\begin{tabular}{|c|c|}
\hline \multicolumn{2}{|l|}{ Outcome variable } \\
\hline HAPU prevalence & - Categories I-IV \\
\hline \multicolumn{2}{|c|}{ Independent variables } \\
\hline $\begin{array}{l}\text { Organisational } \\
\text { variables }\end{array}$ & $\begin{array}{l}\text { Teamwork mean score (0-100) } \\
\text { Safety climate mean score } \\
\text { (0-100) } \\
\text { Perception of management mean } \\
\text { score (0-100) } \\
\text { Ward type (surgery/internal } \\
\text { medicine, rehabilitation, ICU) } \\
\text { Patient/nurse ratio (number of } \\
\text { patients per nurse) } \\
\text { Repositioning (no/yes) } \\
\text { Support surfaces (no/yes) } \\
\text { - Elevated heels (no/yes) }\end{array}$ \\
\hline Patient variables & $\begin{array}{l}\text { Gender } \\
\text { Age }(<70 / \geq 70) \\
\text { Braden total score }(6-23) \\
\text { BMI }(<18.5,18.5-24.99 \\
\left.25-29.99,>30 \mathrm{~kg} / \mathrm{m}^{2}\right)\end{array}$ \\
\hline
\end{tabular}


The organisational variables were ward type, patient-to-nurse ratio (number of patient beds on the ward/number of nurses on the day shift), PU prevention implemented and ward patient safety culture. The PU prevention implemented variable was based on three items: repositioning (no planned, every 2, 3 and $4 \mathrm{~h}$ ), support surfaces (standard mattress, non-powered or powered redistributing mattress) and elevated heels (no/yes). Since PU prevention is dependent on the availability of pressure-redistributing mattresses and health personnel for repositioning, we defined PU prevention implemented as an organisational variable. Patient safety culture was measured by the Safety Attitudes Questionnaire (SAQ). The SAQ has been translated into Norwegian and has been found to have satisfactory psychometric properties in the Norwegian hospital setting. ${ }^{22}$ SAQ measures 36 items in six dimensions: teamwork (6), safety climate (7), perceptions of management (10), job satisfaction (5), stress recognition (4) and working conditions (4). ${ }^{22}{ }^{23}$ The national patient safety culture study used only the first three dimensions from the SAQ to measure patient safety culture. Teamwork measures the perceived quality of collaboration between personnel. Safety climate measures the perceptions of a strong and proactive organisational commitment to safety. Perception of management measures approval of managerial action. ${ }^{23}$ Only three items from this dimension were used in the Norwegian patient safety study. In the SAQ data reports, negatively worded sentences were recoded. Moreover, scores for each item and mean score were converted from a 5-point Likert scale to a 100 -point scale with 0 points indicating the most negative score and 100 the most positive. Staff mean scores were used to characterise the patient safety culture of the wards. Higher scores indicate stronger safety-mindedness. For one hospital that only provided department-level data, the department mean score was used in lieu of ward-level data.

Patient background characteristic variables included gender, age, Braden total score and body mass index (BMI), which have all been found to be significant predictor variables in earlier studies. ${ }^{9} 11$ The Braden scale has six subscales (sensory perception, moisture, activity, mobility, nutrition, friction/shear); each subscale ranges from 1 (worst) to 4 (best), except the friction/shear subscale, which is rated from 1 to 3 . The Braden total score thus ranges from 6 to 23 , where a lower total score means higher risk. ${ }^{24}$

\section{Statistical analysis}

Analysis was conducted by using SPSS (V.21). Missing data on repositioning and pressure redistributing support surfaces were interpreted as no planned repositioning and no pressure redistributing support surfaces. The variable $P U$ prevention implemented was constructed using the three items: mattress, repositioning and elevated heels. The items were first dichotomised to indicate whether the preventive measure was implemented or not. The three items were then summed and the sum score $(0-3)$ was then dichotomised using the cut point $0=$ preventive measures not implemented $/$ score $1-3=$ preventive measures implemented. The sum of the three SAQ dimensions was divided by three and labelled patient safety culture mean score. The patient safety culture constructs Cronbach's coefficient $\alpha$ was 0.905 . We checked for multicollinearity between the predictor variables and none correlated above 0.50 .

Owing to the hierarchical structure of the data, the assumption of independence of observations may not hold, thus requiring multilevel analysis. ${ }^{25}$ It has been argued that even an intraclass correlation coefficient (ICC) as small as $1 \%$ may have design effects that should not be ignored, ${ }^{26}$ and most statisticians agree that an ICC of $10 \%$ or higher calls for multilevel analysis. ${ }^{27}$ Our ICC result was higher than $10 \%$, and we therefore conducted multilevel analysis by MLwiN 2.30. With an MLwiN multilevel logistic regression, the patient-level variance does not automatically appear and we used $\pi^{2} / 3$ for this estimation, as suggested by Rabe-Hesketh and Skrondal ${ }^{28}$ and Twisk ${ }^{29}$. We applied a two-level model (ward and patient levels) due to the limited number of participating hospitals; four hospitals were too few for a model including a hospital level. ${ }^{27} 30{ }^{31}$ Further, we found no hospital-level variance in the PU prevalence study. ${ }^{5}$ The level of significance was set to $\mathrm{p}<0.05$.

To determine how much of the variance in the odds of HAPU was at ward level, that is, across wards, we first applied an empty model, a model with no explanatory variables. ${ }^{32}{ }^{33}$ We then added organisational variables to the model to investigate the association with HAPU. Finally, we included the patient-related risk factors in the model.

\section{Ethics}

All participating patients or relatives received oral and written information and gave verbal consent to participate. The patient safety culture study was a part of a national campaign for which each RHA was legally responsible. Both studies have been conducted in accordance with the Declaration of Helsinki.

\section{RESULTS}

Most participating wards were surgery or internal medicine. The HAPU prevalence was highest for ICU wards and lowest for rehabilitation wards (table 2).

Variable scores differed by ward type (table 3). The highest patient safety culture mean score was found in ICU wards. The patient safety culture mean score ranged from a low score of 52.7 in one rehabilitation ward to the highest score of 81.3 measured for one ward within the surgery and internal medicine group. For the single dimensions, perception of management was lower than teamwork and safety climate. ICU wards had the lowest patient-to-nurse ratio and a higher use of preventive measures than the other two ward types. Likewise, 
Table 2 Patients included and prevalence of HAPU (categories I-IV) by ward type ( $\mathrm{N}=1056)$

\begin{tabular}{lllrr}
\hline & $\begin{array}{l}\text { Surgery, internal medicine } \\
\mathbf{n = 6 2}\end{array}$ & $\begin{array}{l}\text { ICU* } \\
\mathbf{n = 1 5}\end{array}$ & $\begin{array}{l}\text { Rehabilitation } \\
\mathbf{n = 7}\end{array}$ & $\begin{array}{c}\text { Total } \\
\mathbf{N = 8 4}\end{array}$ \\
\hline Patients included ( $(\%))$ & $892(84.5)$ & $76(7.2)$ & $88(8.3)$ & $1056(100)$ \\
HAPU categories I-IV (n (\%)) & $125(14.0)$ & $21(27.6)$ & $5(5.7)$ & $151(14.3)$ \\
\hline${ }^{* B}$ Both postanaesthesia recovery wards and ICUs. & & \\
HAPU, hospital-acquired pressure ulcers; ICU, intensive care unit. & &
\end{tabular}

the patients in ICU wards had the lowest Braden total score indicating higher risk patients. The number of patients 70 years or above was highest in the surgery and internal medicine wards.

The multilevel analysis produced an ICC at ward level above $20 \%$ for HAPUs in the model with no explanatory variables (table 4). When controlled for organisational variables, the average ward patient safety culture score was significantly related to the HAPU odds: one single point up on the $0-100$ patient safety scale was associated with a reduction in the odds by a factor of 0.98 . The odds of HAPU for patients in rehabilitation wards were almost one-fourth of the odds of the reference type of ward. There were no significant differences in the odds of HAPU between ICUs and the reference type of ward; nor was the patient-to-nurse ratio significantly associated with HAPU. When PU prevention was implemented, patients had almost four times higher odds of HAPU as patients who were not allocated any PU prevention. The model with ward-level variables only did not eliminate the across-ward variation in HAPU odds.

The addition of the patient variables (gender, age, Braden total score and BMI) did not affect the significance and the direction of the effects of the organisational variables. Moreover, the associations between
HAPU odds and hospitalisation on a rehabilitation ward and better ward patient safety culture, respectively, were actually strengthened. The association of HAPU odds with PU preventive measures was weakened. However, the odds of HAPU were still twice as high in cases where PU preventive measures had been applied.

When controlled for the other variables in the final model, age was significantly related to HAPU. Patients above 70 years of age had almost three times as high odds of developing an HAPU compared with younger patients. Moreover, the Braden total score was a significant HAPU predictor: one single Braden point reduced the HAPU odds by a factor of as much as 0.73 . The somewhat overweight patient had significantly lower HAPU odds. The other BMI groups did not differ significantly from the reference BMI group. Gender was not significantly related to the odds of developing PU during hospitalisation. Further, in the final model, there was no longer a significant across-ward variance in HAPU odds.

On the basis of the findings for implemented preventive measures, we conducted an additional analysis based on the patient's risk level (Braden score below 17 and/ or a PU). The Braden total score was calculated for 1004 patients, and 222 patients $(22.1 \%)$ were considered at

Table 3 Descriptive statistics for organisational and patient variables $(\mathrm{N}=1056)$

\begin{tabular}{|c|c|c|c|c|}
\hline & $\begin{array}{l}\text { Surgery, } \\
\text { internal medicine }\end{array}$ & ICU* & Rehabilitation & Total \\
\hline \multicolumn{5}{|l|}{ Organisational variables } \\
\hline Patient safety culture mean score (mean(SD)) $(n=1042)$ & $70.1(5.1)$ & $71.6(3.6)$ & $68.5(9.5)$ & $70.1(5.6)$ \\
\hline Teamwork & $75.8(5.4)$ & $77.3(3.9)$ & $74.1(9.9)$ & $75.8(5.9)$ \\
\hline Safety climate & $73.7(5.4)$ & $76.9(4.0)$ & $70.4(7.6)$ & $73.6(5.6)$ \\
\hline Perception of management & $60.8(6.2)$ & $60.5(3.6)$ & $60.9(9.5)$ & $60.8(6.7)$ \\
\hline Patient/nurse ratio (mean(SD)) $(n=1024)$ & $2.8(0.8)$ & $1.2(0.8)$ & $2.6(0.4)$ & $2.6(0.9)$ \\
\hline PU prevention implemented (yes, \%) $(n=1056)$ & 27.4 & 75.0 & 35.2 & 31.4 \\
\hline \multicolumn{5}{|l|}{ Patient variables } \\
\hline Braden total score (mean(SD)) $(n=1004)$ & $20.2(3.1)$ & $16.8(4.5)$ & $19.6(2.7)$ & 19.9 (3.3) \\
\hline Gender (female, \%) $(n=1031)$ & 47.9 & 34.2 & 28.7 & 45.3 \\
\hline Age $(>70$ years, \%) $(n=1045)$ & 40.8 & 26.0 & 21.6 & 38.2 \\
\hline $\mathrm{BMI}, \mathrm{kg} / \mathrm{m}^{2}(\mathrm{n}(\%))$ & 707 (100) & $65(100)$ & $87(100)$ & $859(100)$ \\
\hline Underweight $(<18.5)$ & $41(5.8)$ & $4(6.2)$ & $6(6.9)$ & $51(5.9)$ \\
\hline Normal (18.5-24.99) & $320(45.3)$ & $24(36.9)$ & $48(55.2)$ & $392(45.6)$ \\
\hline Overweight (25-29.99) & $243(34.4)$ & $25(38.5)$ & $24(27.6)$ & $292(34.0)$ \\
\hline Obesity $(>30)$ & $103(14.6)$ & $12(18.5)$ & $9(10.3)$ & $124(14.4)$ \\
\hline
\end{tabular}

${ }^{\star}$ Both postanaesthesia recovery wards and ICUs.

$\mathrm{BMI}$, body mass index; ICU, intensive care unit; PU, pressure ulcer. 
Table 4 Multilevel models with organisational and patient variables associated with HAPU (N=1056)

\begin{tabular}{|c|c|c|c|}
\hline Risk factors & $\begin{array}{l}\text { Empty } \\
\text { model } \\
\mathrm{N}=1056\end{array}$ & $\begin{array}{l}\text { Organisational } \\
\text { variables } \\
n=1010 \\
\text { OR }(95 \% \mathrm{Cl})\end{array}$ & $\begin{array}{l}\text { Organisational and } \\
\text { patient variables } \\
\mathrm{n}=757 \\
\text { OR }(95 \% \mathrm{Cl})\end{array}$ \\
\hline Patient safety culture mean score & & 0.98 (0.96 to 0.99$)$ & $0.97(0.96$ to 0.99$)$ \\
\hline \multicolumn{4}{|c|}{ Ward type (reference group: surgery and internal medicine) } \\
\hline $\mathrm{ICU}$ & & 1.19 (0.43 to 3.33$)$ & $1.14(0.33$ to 3.96$)$ \\
\hline Rehabilitation & & 0.26 (0.08 to 0.87$)$ & 0.17 (0.04 to 0.66$)$ \\
\hline Patient/nurse ratio & & $0.82(0.56$ to 1.21$)$ & 0.99 (0.63 to 1.54$)$ \\
\hline PU prevention implemented (reference group: no) & & 3.74 (2.49 to 5.63$)$ & $2.02(1.12$ to 3.64$)$ \\
\hline Gender (reference group: female) & & & 0.97 (0.57 to 1.65$)$ \\
\hline Age (reference group: <70 years) & & & 2.70 (1.54 to 4.74$)$ \\
\hline Braden scale total score & & & $0.73(0.67$ to 0.80$)$ \\
\hline \multicolumn{4}{|l|}{ BMI (reference group: normal 18.5-24.99 kg/m²) } \\
\hline Underweight & & & $1.46(0.61$ to 3.46$)$ \\
\hline Overweight & & & $0.32(0.17$ to 0.62$)$ \\
\hline Obesity & & & 0.51 (0.22 to 1.18$)$ \\
\hline ICC (\%) & 21.16 & 17.39 & 10.60 \\
\hline
\end{tabular}

risk; of those, $136(61.3 \%)$ received preventive measures compared with $181(23.1 \%)$ for those considered not at risk $\left(\chi^{2}=116.27, \mathrm{p}>0.000\right)$.

\section{DISCUSSION}

The multilevel analysis found significant associations between HAPU odds and both organisational and patient variables. One finding in our study was the significant association between the patient safety culture score and the presence of HAPU: the higher the patient safety culture score, the lower the HAPU odds. An OR of 0.97 means a 10 -point increase in patient safety score result would correspond to a $26 \%\left(1-0.97^{10}\right)$ reduction in HAPU odds. Ward patient safety scores in the data set varied by as much as 28,7 points, from a low of 52.7 to a high of 81.3. This significant association between higher patient safety and lower odds of HAPU echoes earlier studies that found an association between global safety score, safety climate and team work, and the odds of PU. ${ }^{12} 13$ On the other hand, other researchers have reported no association between organisational culture, team climate, preventive quality management at the ward level and safety culture climate and the presence of PU. ${ }^{14} 15$ This study supports a significant association between patient safety culture and PU prevalence; however, further research is warranted to conclude with greater certainty.

Further, the rehabilitation wards had significantly lower odds for HAPUs than the surgery and internal medicine wards. Additional analysis showed that onefifth of the patients in the rehabilitation wards were considered to be at risk and/or had PU (data not shown). The patients at rehabilitation wards are probably more mobilised and in a healthier state than the hospitalised patients in surgical and internal medicine wards.

One further significant result was the counterintuitive finding that implementation of PU prevention seemed to increase the odds of HAPU. We interpret this to mean that such measures were often implemented after the HAPU had occurred and not solely based on an a priori risk assessment. Further, the additional analysis revealed that patients at risk and/or with a PU were significantly more likely to receive such measures compared with those not at risk. About $60 \%$ of those at risk and/or with a PU had preventive measures implemented. The international guideline recommends that all at-risk patients should have preventive measures. ${ }^{11}$ For those not at risk, about one-fourth had preventive measures. However, we do not know if these patients had been considered at risk prior to the data collection day and not been reassessed as not at risk. It may also be argued that some patients were allocated PU prevention unnecessarily. This practice may be questioned considering that prevention also is costly, an issue also raised by Vanderwee et al. ${ }^{34}$ We also do not know whether the preventive measures were implemented as primary or secondary prevention. An ICU study also found a significant association between preventive measures and PU outcome (category II-IV). ${ }^{35}$ The researchers explained that patients were correctly identified as at risk, but preventive measures were applied too late or first after the PU became visible. ${ }^{35}$ Likewise, in our study, the nurses had probably not implemented the international evidence-based guideline that is available in Norwegian. ${ }^{36}$ Assessment of patient risk of compromised skin integrity is a fundamental nursing responsibility. Yet studies have shown that nurses do not give 
prevention the necessary attention and priority to avoid PU from developing. ${ }^{37}$

The single items included in the collapsed preventive measures variable were measured in individual patients, but the collapsed variable was considered to be an organisational variable used as a measurement of nursing care. In a large multicountry study, nurses identified skin care and frequent changing of patient position as nursing activities care left undone. ${ }^{16}$ Moreover, if preventive measures had been considered as a patient variable, the relationship between the significant organisational variables and HAPU would remain (patient safety culture mean score OR 0.94 (95\% CI 0.90 to 0.99 ) and rehabilitation ward OR 0.29 (95\% CI 0.09 to 0.93$)$ ).

The organisational variables were important explanatory factors in the models, but patient variables were also important. Even in wards with a good record of successful prevention of HAPUs, an influx of high-risk patients may affect the HAPU prevalence. Wards have little influence on the number of high-risk patients, but they do have an opportunity to decide the quality of care their patients receive.

These findings raise questions about quality incentives in hospitals. Increased competency among staff in taking preventive measures may reduce the prevalence of HAPU. However, the culture and attitude on the individual wards may influence the implementation of new knowledge and the wards should therefore focus on improved teamwork. Moreover, there is a need for greater focus on safety in clinical practice to protect patients at risk. Finally, the results indicate that nursing staff do not always perceive the management as focusing on safety and quality of care. We need to measure nursing quality outcomes in order to set benchmarks, as these outcomes relate to the organisational quality of care. Moreover, the number of vulnerable patients will increase due to the expected increase in the number of older patients with higher comorbidity and higher patient turnover with shorter length of stay in hospital. It is important to prevent PUs because they affect the individual patient's quality of life as well as increase the cost of care.

\section{Limitations}

A limitation of this study is the use of department-level data for one of the hospitals for the variable mean ward patient safety culture. There were significant differences in the mean ward scores by ward type across the hospitals (data not shown), but this may be due to the range in the number of wards per hospital. We do not know exactly when the PUs in our study occurred: some of the PUs we considered to be acquired in hospitals may have occurred prior to admission, but were not clinically visible at admission. ${ }^{38}$

Further, the limited number of HAPU cases in our data set limited the number of included variables in the multilevel logistic regression models. We adjusted for this limitation by collapsing categories on the variables so that the independent variables could be presented by fewer dummy variables, even though the collapsing of categories results in less information. We also tested for interactions between the variables prior to the model fitting, but none of these were statistically significant (patient safety culturexpreventive measures, patient safety culture $\times$ Braden total score, Braden total score $\times$ age, age $\times$ gender, patient/nurse ratioxpreventive measures, patient/nurse ratioxpatient safety culture, patient safety culturextype of ward).

It would have been interesting to use the same models with the more severe HAPUs, HAPU II-IV, and to check whether the variables that predicted all HAPUs also predicted the more severe HAPUs. Our data set only included 47 patients with HAPUs II-IV (data not shown), and that was too few for the analysis using our set of HAPU variables.

The results that patients with preventive measures had higher odds for HAPU may be due to confounding. In many cases, preventive measures were probably implemented prior to PU development based on PU risk assessment or only after a PU was visible and, moreover, information about the quality and availability of mattresses that may vary from ward to ward. Owing to the study design, we do not have data to assess these potential confounding factors. Future studies should endeavour to further investigate these variables. The preventive measures could also be considered as an intermediate variable between organisational variables and HAPUs. Owing to the sample size, collapsing variables into one variable reduced the amount of information provided, especially preventive measures and ward type. Larger studies are required to enable inclusion of the individual variables in the models.

A cross-sectional study with limited variables is inadequate to demonstrate causality. However, the purpose for our study was to describe the association between selected predictors and HAPU, not a causal relationship.

\section{CONCLUSION}

The fact that the odds of HAPU varied across wards, and that across-ward variance was reduced when the selected ward-level variables entered the explanatory model, indicates that the HAPU problem may be reduced by ward-level organisation of care improvements, that is, by improving the patient safety culture and implementation of preventive measures. Some wards may prevent PU better than other wards. The fact that ward-level variation was eliminated when patient-level HAPU variables were included in the model indicates that even wards with the best HAPU prevention will be challenged by an influx of high-risk patients.

Acknowledgements The authors thank the study coordinators at the participating hospitals and nursing staff at the participating wards.

Contributors IMB has been responsible for conceptual development and design, had the lead on data collection, analysis and interpretation of data, and has drafted and completed the submitted version of the manuscript. 
DH contributed to the data analysis. All coauthors have contributed to the development of the concept and design of the study, interpretation of data and manuscript drafting, as well as provided comments and ideas during the process, and gave their final approval to the final manuscript.

Funding This study was supported financially by Oslo University Hospital, The Norwegian Nurses Organisation, University of Oslo and Sophies Minde Ortopedi AS

\section{Competing interests None declared.}

Ethics approval The privacy protection officer of each participating hospital approved the PU prevalence multicentre study protocol.

Provenance and peer review Not commissioned; externally peer reviewed.

Data sharing statement No additional data are available.

Open Access This is an Open Access article distributed in accordance with the Creative Commons Attribution Non Commercial (CC BY-NC 4.0) license which permits others to distribute, remix, adapt, build upon this work noncommercially, and license their derivative works on different terms, provided the original work is properly cited and the use is non-commercial. See: http:// creativecommons.org/licenses/by-nc/4.0/

\section{REFERENCES}

1. Speroff T, Nwosu S, Greevy R, et al. Organisational culture: variation across hospitals and connection to patient safety climate. Qual Saf Health Care 2010;19:592-6.

2. Norwegian Ministry of Health and Care Services. Meld. St.16 (2010 2011) Report to the Storting (white paper)-National Health and Care Services Plan (2011-2015). Norwegian Ministry of Health and Care Services, 2011.

3. Health Safety Commission. Third Report: Organising for Safety, ACSNI Study Group on Human Factors. London: HMSO, 1993.

4. Halligan M, Zecevic A. Safety culture in healthcare: a review of concepts, dimensions, measures and progress. BMJ Qual Saf 2011;20:338-43.

5. Bredesen IM, Bjoro K, Gunningberg L, et al. The prevalence, prevention and multilevel variance of pressure ulcers in Norwegian hospitals: a cross-sectional study. Int J Nurs Stud 2015;52:149-56.

6. OECD. Health at a Glance 2011. OECD Publishing, 2011.

7. Aiken LH, Sloane DM, Bruyneel L, et al. Nurses reports of working conditions and hospital quality of care in 12 countries in Europe. Int J Nurs Stud 2013;50:143-53.

8. Tvedt C, Sjetne IS, Helgeland J, et al. A cross-sectional study to identify organisational processes associated with nurse-reported quality and patient safety. BMJ Open 2012;2:pii:e001967. doi:10 1136/bmjopen-2012-001967

9. Coleman S, Gorecki C, Nelson EA, et al. Patient risk factors for pressure ulcer development: systematic review. Int J Nurs Stud 2013:50:974-1003.

10. Dealey C, Brindle CT, Black J, et al. Challenges in pressure ulcer prevention. Int Wound J 2015;12:309-12.

11. National Pressure Ulcer Advisory Panel, European Pressure Ulcer Advisory Panel, Pan Pacific Pressure Injury Alliance. Prevention and treatment of pressure ulcers: clinical practice guideline. Perth, Australia: Cambridge Media, 2014.

12. Taylor JA, Dominici F, Agnew J, et al. Do nurse and patient injuries share common antecedents? An analysis of associations with safety climate and working conditions. BMJ Qual Saf 2012;21:101-11.

13. Brown DS, Wolosin R. Safety culture relationships with hospital nursing sensitive metrics. J Healthc Qual 2013;35:61-74.

14. Bosch M, Halfens RJ, van der Weijden T, et al. Organizational culture, team climate, and quality management in an important patient safety issue: nosocomial pressure ulcers. Worldviews Evid Based Nurs 2011;8:4-14.
15. Ausserhofer D, Schubert M, Desmedt M, et al. The association of patient safety climate and nurse-related organizational factors with selected patient outcomes: a cross-sectional survey. Int J Nurs Stud 2013;50:240-52.

16. Ausserhofer D, Zander B, Busse R, et al. Prevalence, patterns and predictors of nursing care left undone in European hospitals: results from the multicountry cross-sectional RN4CAST study. BMJ Qual Saf 2014;23:126-35.

17. Sullivan N, Schoelles KM. Preventing in-facility pressure ulcers as a patient safety strategy: a systematic review. Ann Intern Med 2013;158(5 Pt 2):410-16.

18. Hearld LR, Alexander JA, Fraser I, et al. Review: how do hospital organizational structure and processes affect quality of care?: a critical review of research methods. Med Care Res Rev 2008;65:259-99.

19. Norwegian Ministry of Health and Care Services. Meld.St.10 (20122013) Report to the Storting (white paper)-High Quality - Safe services. Norwegian Ministry of Health and Care Services, 2012

20. Nasjonalt kunnskapssenter for helsetjenester. In safe hands. 2014 http://www.pasientsikkerhetsprogrammet.no/no/l+trygge+hender/ln +English (accessed 5 Jun 2014).

21. Bours GJ, Halfens RJ, Abu-Saad HH, et al. Prevalence, prevention, and treatment of pressure ulcers: descriptive study in 89 institutions in the Netherlands. Res Nurs Health 2002;25:99-110.

22. Deilkas ET, Hofoss D. Psychometric properties of the Norwegian version of the Safety Attitudes Questionnaire (SAQ), Generic version (Short Form 2006). BMC Health Serv Res 2008;8:191.

23. Sexton JB, Helmreich RL, Neilands TB, et al. The Safety Attitudes Questionnaire: psychometric properties, benchmarking data, and emerging research. BMC Health Serv Res 2006;6:44.

24. Bergstrom N, Demuth PJ, Braden BJ. A clinical trial of the Braden scale for predicting pressure sore risk. Nurs Clin North Am 1987;22:417-28.

25. Leyland A, Goldstein H. Multilevel modelling of health statistics. Chichester: Wiley, 2001.

26. Gulliford MC, Ukoumunne OC, Chinn S. Components of variance and intraclass correlations for the design of community-based surveys and intervention studies: data from the Health Survey for England 1994. Am J Epidemiol 1999;149:876-83.

27. Kahn JH. Multilevel modeling: overview and applications to research in counseling psychology. J Couns Psychol 2011;58:257-71.

28. Rabe-Hesketh S, Skrondal A. Multilevel and longitudinal modeling using Stata. College Station, Texas: Stata Press, 2012.

29. Twisk JWR. Applied multilevel analysis: a practical guide. Cambridge: Cambridge University Press, 2006.

30. Nezlek JB. An introduction to multilevel modeling for social and personality psychology. Soc Personal Psychol Compass 2008:2:842-60

31. Blance A. Multilevel modelling. In: Ty YK, Greenwood DC, eds Modern methods for epidemiology. Dordrecht: Springer Science +Business Media, 2012:73-91.

32. Cress U. The need for considering multilevel analysis in CSCL research-an appeal for the use of more advanced statistical methods. Comput Support Learn 2008;3:69-84.

33. Hox JJ. Multilevel analysis: techniques and applications. New York: Routledge, 2010.

34. Vanderwee K, Defloor T, Beeckman D, et al. Assessing the adequacy of pressure ulcer prevention in hospitals: a nationwide prevalence survey. BMJ Qual Saf 2011;20:260-7.

35. Nijs N, Toppets A, Defloor T, et al. Incidence and risk factors for pressure ulcers in the intensive care unit. J Clin Nurs 2009;18:1258-66.

36. National Pressure Ulcer Advisory Panel, European Pressure Ulcer Advisory Panel. Forebygging av trykksår. Kortutgave av retningslinjen. 2012. http://www.epuap.org/guidelines/QRG Prevention_in_Norwegian.pdf

37. Sving E, Gunningberg L, Hogman M, et al. Registered nurses' attention to and perceptions of pressure ulcer prevention in hospital settings. J Clin Nurs 2012;21:1293-303.

38. Brown DS, Aydin CE, Donaldson N, et al. Benchmarking for small hospitals: size didn't matter! J Healthc Qual 2010;32:50-60. 